\title{
Gewerkschaftliche Strategien in der Leiharbeit
}

Die fortlaufende Prekarisierung von Beschäftigungsverhältnissen bedeutet für die deutschen Gewerkschaften eine massive Herausforderung. Wie reagieren die Gewerkschaften auf die Ausweitung unsicherer, niedrig entlohnter Beschäftigung und die damit verbundenen Auswirkungen? Welche Strategien werden gewählt und wie sind diese zu verorten? Diesen Fragen soll im Folgenden am Beispiel der Leiharbeit im Organisationsbereich der IG Metall nachgegangen werden.

\section{Einleitung}

Die Ausweitung unsicherer, niedrig entlohnter Beschäftigungsverhältnisse ist in letzter Zeit unter dem Begriff der Prekarisierung verstärkt in die Diskussion geraten. Besonders die rasante Expansion der Leiharbeit hat aufgrund der mit diesem Arbeitsverhältnis verbundenen nachteiligen Arbeitsbedingungen hohe Aufmerksamkeit auf sich gezogen.

Nach dem aktuellen DGB-Index „Gute Arbeit" bewerten Leiharbeitnehmer ihre Arbeit deutlich schlechter als regulär, aber auch als befristet Beschäftigte. Die schlechtere Bewertung bezieht sich dabei nicht nur auf die Arbeitsplatzsicherheit und Einkommenssituation, sondern auch auf Aufstiegsmöglichkeiten, Qualifizierungs- und Entwicklungsmöglichkeiten sowie die körperlichen Anforderungen.

Mit einem Anteil von ca. 1,5 \% an allen Beschäftigungsverhältnissen hat die Leiharbeit zwar quantitativ einen immer noch vergleichsweise geringen Stellenwert, allerdings ist die Entwicklung dieser Beschäftigungsform hoch dynamisch: Nach Angaben der Bundesagentur für Arbeit hat sich die Zahl der Leiharbeitsverhältnisse von 327.000 im Jahr 2003 bis Ende 2006 auf über 631.000 fast verdoppelt (Bundesagentur für Arbeit 2007; vgl. auch Abbildung 1).

Besonders betroffen von dieser Entwicklung ist der Organisationsbereich der IG Metall. Mehr als die Hälfte der Leiharbeitnehmer wird in der Metall- und Elektroindustrie eingesetzt. Bezogen auf die Gesamtzahl der im Organisationsbereich der IG Metall beschäftigten Arbeitnehmer arbeitet bereits jeder Zehnte in einem Leiharbeitsverhältnis (Wassermann/Rudolph 2007, S. 6).

Diese Entwicklung stellt die Gewerkschaft vor mehrere Aufgaben: Erstens ent-

Abb. 1: Bestand an Leiharbeitnehmern (jeweils Ende Dezember)

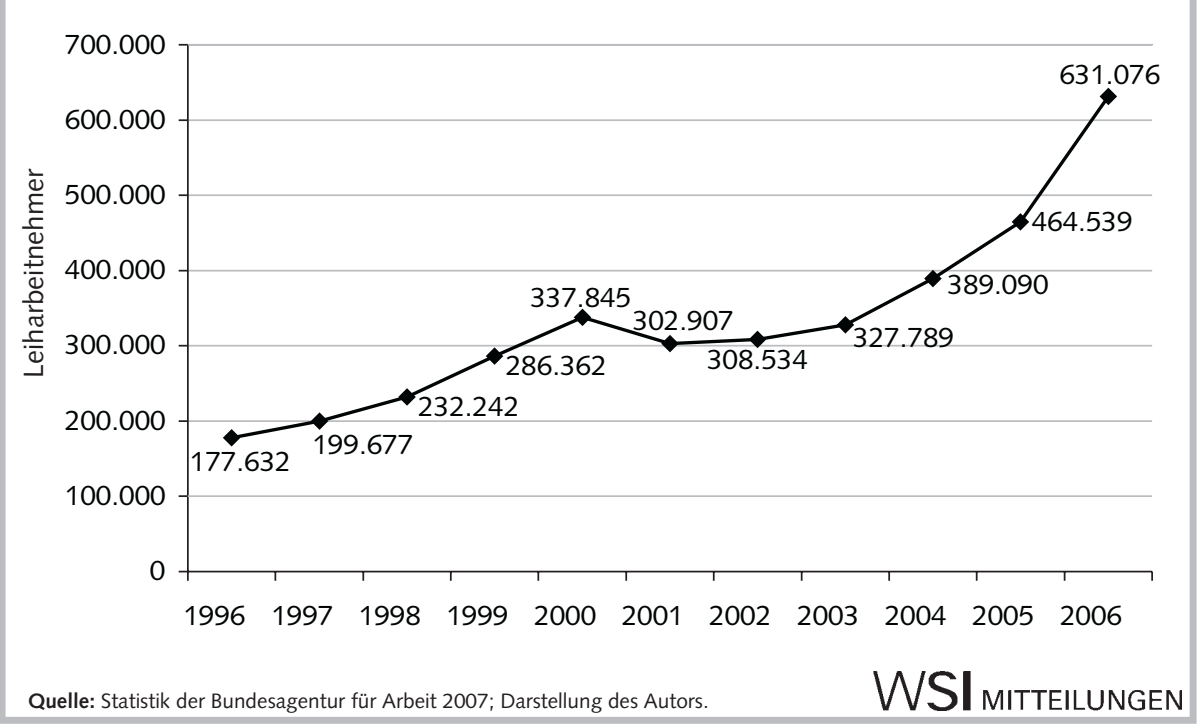

steht die Gefahr einer Verschärfung der Mitgliederkrise, weil sie traditionell auf diese Beschäftigtengruppe nicht ausgerichtet ist und Schwierigkeiten hat, die Leiharbeitnehmer zu organisieren (Aust/Holst 2006). Hieraus resultiert die Herausforderung, die Leiharbeitnehmer zu repräsentieren und zu organisieren.

Zweitens bedroht die Ausweitung unorganisierter, prekärer Beschäftigungsverhältnisse die erreichten gewerkschaftlichen Arbeitsstandards der regulär beschäftigten Arbeitnehmer und führt zu einer Verunsicherung der organisierten Stammbelegschaften (Dörre et al. 2004). Hieraus ergibt sich für die Gewerkschaften die Herausforderung, die prekären Beschäftigungsverhältnisse zu regulieren und einzugrenzen.

Vor dem Hintergrund dieser Problemstellung soll in diesem Artikel folgenden Fragen nachgegangen werden: Wie reagieren Gewerkschaften auf die Ausweitung prekärer Beschäftigung? Welche Strategien werden gewählt und wie sind diese zu verorten?

Diese Fragen sollen anhand der Analyse gewerkschaftlicher Strategien gegenüber der prekären Beschäftigungsform Leiharbeit im Organisationsbereich der IG Metall beantwortet werden.

Die folgenden Ausführungen reflektieren erste Ergebnisse einer Forschungsarbeit zum Thema gewerkschaftliche Organisierung von Leiharbeitnehmern und basieren auf leitfadengestützten Interviews mit Betriebsräten aus Ent- und Verleihbetrieben sowie mit ehren- und hauptamtlichen Gewerkschaftern. ${ }^{1}$

\footnotetext{
1 Bei dem Projekt "Gewerkschaftliche Mitgliedergewinnung in der Leiharbeit" handelt es sich um das Promotionsvorhaben des Autors. Die Auswertungen stützen sich auf bislang insgesamt 25 durchgeführte Interviews, davon sechs mit Betriebsräten von Verleihfirmen.
}

Tobias Wölfle, Sozialwissenschaftler promoviert an der Friedrich-SchillerUniversität Jena, Fachbereich Soziologie, über gewerkschaftliche Organisierung in der Leiharbeit. Arbeitsschwerpunkt: Arbeitssoziologie e-mail: tobwoel@web.de 


\section{Entwicklung der Leiharbeit bis 2002}

Leiharbeit wurde in der Bundesrepublik erst 1967 legalisiert und hatte auch danach zunächst keine große Bedeutung für den Arbeitsmarkt. Gründe dafür waren die geringe gesellschaftliche Akzeptanz von Leiharbeit sowie die relativ strenge Regulierung dieser Beschäftigungsform durch das Arbeitnehmerüberlassungsgesetz (AÜG) von 1972. Die darin enthaltenen Regelungen sollten vor allem eine kontinuierliche Beschäftigung des Leiharbeitnehmers gewährleisten und damit eine Abwälzung des Risikos einsatzfreier Zeiten auf die Arbeitnehmer verhindern. $\mathrm{Zu}$ diesem Zweck beinhaltete das AÜG ein Synchronisationsverbot, das zum einen untersagte, die Beschäftigungsdauer des Leiharbeitnehmers im Verleihbetrieb auf die Einsatzzeit im Entleihbetrieb zu beschränken, zum anderen enthielt es ein Wiedereinstellungsverbot, um wiederholte kurztaktige Beschäftigungseinsätze zu vermeiden, und ein besonderes Befristungsverbot.

Weiterhin sollten durch eine gesetzliche Überlassungshöchstdauer, d.h. durch eine zeitliche Begrenzung des Verleihs an einen Entleiher, Verdrängungseffekte in den Einsatzbetrieben unterbunden werden.

In der Praxis wurden die Bestimmungen zur Beschäftigungssicherung von den Zeitarbeitsfirmen allerdings häufig missachtet, um eine gesetzlich vorgeschriebene Entlohnung der Leiharbeitnehmer in den einsatzfreien Zeiten zu umgehen (Schüren 2005, S.13). Zudem wurde die Überlassungshöchstdauer ab 1985 mit der politischen Zielsetzung einer Flexibilisierung des Arbeitsmarktes mehrfach verlängert, um auch längerfristige Einsätze von Leiharbeitnehmern zu ermöglichen.

Diese Deregulierung des AÜG begünstigte das schnelle Wachstum der Leiharbeit in den 1990er Jahren. Daneben wurde die Ausweitung der Leiharbeit aber auch durch die zunehmend größer werdende Lohndifferenz zwischen Leiharbeitnehmern und regulär Beschäftigten befördert. So wuchs der Abstand der Einkommen der Leiharbeitnehmer zu den gesamtwirtschaftlichen Durchschnittseinkommen infolge der Massenarbeitslosigkeit von 28,3\% im Jahre 1990 auf 41,2 \% im Jahre 2001 (Rudolph 2003, S. 23).

\section{Frühe gewerkschaftliche Strategien in der Leiharbeit}

Aufgrund der prekären Konstellation des Leiharbeitsverhältnisses und der befürchteten negativen Auswirkungen dieses Arbeitsverhältnisses auf die erreichten $\mathrm{Be}$ schäftigungsstandards der regulär beschäftigten Arbeitnehmerschaft standen die Gewerkschaften der Leiharbeit lange Zeit ablehnend gegenüber. Ausdruck dieser Haltung war die im DGB-Grundsatzprogramm von 1981 enthaltene Forderung nach einem Verbot der Leiharbeit.

Allerdings wurde die Verbotsforderung in den 1990er Jahren zunehmend infrage gestellt und 1996 aus dem Grundsatzprogramm des DGB gestrichen. Stattdessen erklärte der DGB 1998 die Absicht, eine Regulierung der Leiharbeit über Tarifverträge zu erreichen.

Ein Grund für diesen Strategiewandel war die politische Entwicklung, die ganz im Gegensatz zur Verbotsforderung den Einsatz von Leiharbeit beförderte, sodass die Aussicht auf ein Verbot der Leiharbeit zunehmend unrealistisch erschien. Ein zweiter Grund lag in der stetig wachsenden Bedeutung dieser Beschäftigungsform, die von den Gewerkschaften ein aktiveres Handlungskonzept gegenüber der Leiharbeit erforderte. Daneben wurde der Strategiewandel aber auch durch eine zunehmend positive Bewertung der Leiharbeit als arbeitsmarktpolitisches Instrument befördert. Vor dem Hintergrund wachsender Arbeitslosigkeit und sich verfestigender Langzeitarbeitslosigkeit galt die Leiharbeit auch den Gewerkschaften als mögliche Brücke in den Arbeitsmarkt. So beteiligte sich der DGB in Nordrhein-Westfalen bereits 1995 an der Gründung der vom Land initiierten Verleihfirma START, über die Arbeitslose in dauerhafte Beschäftigung vermittelt werden sollten.

Im Zuge dieses Strategiewandels schloss die IG Metall Ende der 1990er Jahre erste Tarifverträge mit Zeitarbeitsfirmen, in deren Folge auch gewerkschaftlich organisierte Betriebsräte gegründet wurden. Begünstigend wirkte dabei, dass sich einige Verleihfirmen von Tarifabschlüssen einen erleichterten Zugang zu gewerkschaftlich organisierten Entleihfirmen erhofften und sich dementsprechend offen gegenüber den gewerkschaftlichen Ansätzen zeigten. Allerdings blieben diese Tarifierungs- und Organisierungsaktivitäten sowohl hinsichtlich der betreffenden Zeitarbeitsfirmen als auch regional begrenzt.

\section{4 \\ Die Tarifierung der Leiharbeit 2003}

$\mathrm{Zu}$ einem umfassenden Wandel der gewerkschaftlichen Haltung gegenüber der Leiharbeit kam es im Zuge der von der Hartz-Kommission angeregten Neugestaltung des AÜG im Jahre 2002. Diese hatte unter Beteiligung von Vertretern von ver.di und der IG Metall ein neues Leitbild für die Arbeitnehmerüberlassung entwickelt, in dem einer gesetzlich deregulierten Leiharbeit als flexibler Beschäftigungsform eine zentrale Rolle beim Abbau der Arbeitslosigkeit zukommen sollte (Kommission Moderne Dienstleistungen am Arbeitsmarkt 2002, S.157). Die Deregulierung sollte allerdings durch eine umfassende tarifliche Regulierung der Leiharbeit flankiert werden und so die Anerkennung der Leiharbeit durch die Gewerkschaften sichern.

Die im Dezember 2002 erfolgte gesetzliche Neugestaltung der Leiharbeit beinhaltete die Abschaffung der Regulierungen des AÜG einschließlich der Überlassungshöchstdauer. Im Gegenzug wurde - entsprechend einer erwarteten EU-Richtlinie - die Einführung eines Gleichbehandlungsgrundsatzes in das Gesetz aufgenommen, nach dem für Leiharbeitnehmer die gleichen Entlohnungs- und Arbeitsbedingungen wie für vergleichbare Stammarbeitskräfte in den Entleihbetrieben gelten sollten. Allerdings wurde dieser Gleichbehandlungsgrundsatz durch einen Tarifvorbehalt relativiert, der eine Unterschreitung des Gleichstellungsprinzips durch tarifvertragliche Regelungen ermöglichte (Ulber 2004, S. 132f.).

Vor dem Hintergrund der neuen gesetzlichen Situation drängten die Arbeitgeber der Zeitarbeitsbranche auf den Abschluss von Tarifverträgen, um die Geltung des Gleichbehandlungsgrundsatzes zu umgehen. Darauf aufbauend ergab sich für die Gewerkschaften - unter Inkaufnahme einer Abweichung vom gesetzlichen Gleichstellungsprinzip - die Möglichkeit einer umfassenden Tarifierung der Branche. 
Nach ersten Sondierungsgesprächen im Dezember 2002 erklärten sich die DGB-Einzelgewerkschaften im Rahmen einer Tarifgemeinschaft unter Federführung des DGB zu Verhandlungen mit den Arbeitgebern der Zeitarbeitsbranche über Flächentarifverträge zur Leiharbeit bereit. Die scheinbar günstige Verhandlungsposition wurde aber bereits kurz nach Aufnahme zentraler Tarifverhandlungen durch einen Tarifabschluss des christlichen Gewerkschaftsbundes mit einem kleinen Arbeitgeberverband der Zeitarbeitsbranche entwertet, der ein niedriges Tarifniveau fixierte und damit das Tarifierungsmonopol der DGB-Gewerkschaften aufhob.

Trotz der daraus resultierenden Verschlechterung der Verhandlungsposition der DGB-Tarifgemeinschaft kam es 2003 zu Tarifabschlüssen mit den beiden großen Arbeitgeberverbänden der Zeitarbeitsbranche. Diese beinhalteten in der Eingangstufe einen Stundenlohn von $6,85 €$ und in der höchsten Stufe von 15,43€ bzw. $15,50 €$. Für Leiharbeitnehmer in Ostdeutschland wurde dabei ein Abschlag von 13,5\% vereinbart. Der Abschluss der Flächentarifverträge wurde von Seiten des DGB wie auch der Einzelgewerkschaften als ein "großer Erfolg“ gewertet (DGB 2003, S. 5).

Entgegen dieser positiven Bewertung spiegelte der Tarifvertrag weitgehend die organisatorische Schwäche der Gewerkschaften in der Leiharbeit wider: Nach einer Studie des IAT liegen die tariflichen Einstiegslöhne in Westdeutschland ausnahmslos, in Ostdeutschland teilweise unterhalb der Schwelle von $50 \%$ des gesamtwirtschaftlichen Medians und sind damit nach den Kriterien der OECD als „Armutslöhne" zu bezeichnen (Weinkopf 2006, S. 20). Für zahlreiche Leiharbeitnehmer zogen die neuen Flächentarifverträge sogar eine Verschlechterung ihrer Einkommenssituation nach sich. Besonders Beschäftigte aus bereits tarifgebundenen Zeitarbeitsfirmen mussten infolge der Anpassung ihrer Haustarifverträge an die neuen DGB-Tarife Einbußen akzeptieren (Aust et al. 2007, S. 247). Zudem zeigten sich bereits kurz nach Abschluss der Flächentarifverträge dessen Auswirkungen auf die Beschäftigungsstandards in anderen Branchen. So drohten etwa die Unternehmen in der Gebäudereinigung, massiv auf Leiharbeit und damit niedrigere Tarife umzusteigen, und erzwangen einen neuen Tarifvertrag mit der IG BAU, der ab 2004 nominale Lohnsenkungen beinhaltete (Schlese/Schramm 2004).

\subsection{PROJEKT ZOOM UND LOKALE ARBEITSKREISE}

Anders als bei ver.di gab es von Seiten der IG Metall auch nach dem Abschluss der Flächentarifverträge keinen ausdrücklichen Organisierungsanspruch bezüglich der Leiharbeit. Erst Ende 2004 wurde auf Initiative der Tarifabteilung der IG Metall Vorstandverwaltung zur Förderung der Mitgliederwerbung in der Leiharbeit das Projekt ZOOM (Zeitarbeitnehmer ohne Organisation machtlos) initiiert.

Zentraler Bestandteil des Projekts ist eine Internetplattform, über die neben Informationsangeboten zur Leiharbeit auch verschiedene Diskussionsforen für Leiharbeitnehmer und gewerkschaftlich Aktive bereitgestellt werden, um auf diese Weise erste gewerkschaftliche Vernetzungs- und Organisierungsprozesse in der Leiharbeit zu unterstützen.

Schon vor der Initiierung des ZOOMProjekts hatten sich in Reaktion auf die wachsende Bedeutung der Leiharbeit im Organisationsbereich der IG Metall erste lokale Arbeitskreise von engagierten Leiharbeitnehmern und Betriebsräten gegründet. Bereits kurz nach Gründung dieser Arbeitskreise fand eine Vernetzung über das ZOOM-Projekt statt, dass die Gründung neuer Arbeitskreise auf der Internetplattform und im Rahmen von Informationsveranstaltungen seitdem aktiv bewirbt.

Ziel der Arbeitskreise ist es, auf lokaler Ebene Austauschprozesse zwischen Betriebsräten aus Ent- und Verleihbetrieben und Leiharbeitnehmern $\mathrm{zu}$ organisieren und gewerkschaftliche Aktivitäten zur Organisierung und Interessenvertretung von Leiharbeitnehmern zu befördern. Die Organisierungsaktivitäten der Arbeitskreise zielen dabei sowohl auf die Verleihbetriebe, indem Leiharbeitern Hilfestellung bei Betriebsratsgründungen in Zeitarbeitsfirmen geboten wird, als auch auf die Einsatzbetriebe. Hier besteht der Ansatzpunkt in der Informierung und Sensibilisierung der Entleihbetriebsräte für Problemstellungen der Leiharbeiternehmer, um sie auf diese Weise bei der Ansprache von Leiharbeitern zu unterstützen.

\subsection{PROBLEME GEWERKSCHAFT- LICHER ORGANISIERUNG IN DER LEIHARBEIT}

Trotz einer wachsenden Zahl von lokalen Arbeitskreisen im Organisationsbereich der IG Metall ist es bisher nicht gelungen, eine bedeutende Zahl von Leiharbeitnehmern zu organisieren. Dies gilt sowohl für die Mitgliederwerbung über die Zeitarbeitsfirmen als auch mit Blick auf die Werbung von Leiharbeitnehmern in den Entleihbetrieben.

Mit Bezug auf eine gewerkschaftliche Organisierung über die Zeitarbeitsfirmen resultiert dieses Organisierungsdefizit primär aus der Struktur des Leiharbeitsverhältnisses. So erschwert die Streuung der Beschäftigten einer Zeitarbeitsfirma auf unterschiedliche sowie häufig wechselnde Einsatzbetriebe nicht nur die Gründung von Betriebsräten, sondern auch die Mitgliederwerbung über bestehende Betriebsräte in der Zeitarbeitsbranche. Daneben wirken aber auch branchenbezogene Faktoren, wie die hohe Fluktuation in den Zeitarbeitsfirmen und die überwiegend gewerkschaftsfeindliche Haltung vieler Arbeitgeber in der Zeitarbeitsbranche, hemmend auf den Organisierungsprozess. Auf der Basis von Interviews mit Leiharbeitnehmern und Verleihbetriebsräten lässt sich ein weiteres Hindernis der Mitgliederwerbung in der häufig ungünstigen Wahrnehmung der Gewerkschaften durch die Leiharbeitnehmer identifizieren. Dieses resultiert zum einen aus der lange Zeit passiven Haltung der Gewerkschaften gegenüber der Leiharbeit, wodurch die Gewerkschaften häufig gar nicht als Interessenvertretung von Leiharbeitern wahrgenommen werden. Zum anderen aber schlagen auch die niedrigen Abschlüsse der Flächentarifverträge in der Verleihbranche, die für viele Leiharbeitnehmer eine Einkommensverschlechterung bedeuten, negativ auf die Gewerkschaften zurück. Besonders problematisch für die Mitgliedergewinnung wirkt sich in diesem Zusammenhang aus, dass gerade viele Zeitarbeitsfirmen mit Betriebsrat von den Einkommensverschlechterungen betroffen waren.

Aber auch über die im Organisationsbereich existierenden Einsatzbetriebe konnte trotz vorhandener gewerkschaftlicher Präsenzstrukturen bisher keine bedeutsame Mitgliederwerbung betrieben werden. Grundsätzlich bestehen hier zwar durch die Kontaktmöglichkeiten am Arbeitsort 
wesentlich bessere strukturelle Bedingungen der Ansprache als in den Verleihbetrieben, allerdings findet eine aktive Interessenvertretung und Kontaktaufnahme nur in einer Minderheit der Einsatzbetriebe statt (Wassermann/Rudolph 2007, S. 17). Für die überwiegend passive Haltung der Betriebsräte lassen sich auf der Grundlage qualitativer Befragungen zwei Einflussfaktoren nennen:

Erstens pflegen zahlreiche Betriebsräte ein eher distanziertes Verhältnis gegenüber den Leiharbeitern, weil auch sie die Leiharbeitnehmer als eine Flexibilitätsreserve sehen, die die Beschäftigungssicherheit der Stammbelegschaft erhöht (Promberger et al. 2006, S. 154). Zweitens erscheint vielen Betriebsräten Leiharbeit als im Rahmen ihrer Handlungsmöglichkeiten nicht beeinflussbar und damit nicht dem Zuständigkeitsbereich des Betriebsrats zugehörig (Wassermann/Rudolph 2007, S. 20).

Beide Einstellungen befördern Exklusion, sodass Erfolge bei der Mitgliedergewinnung sowohl im Entleih- als auch im Verleihbetrieb sehr gering sind.

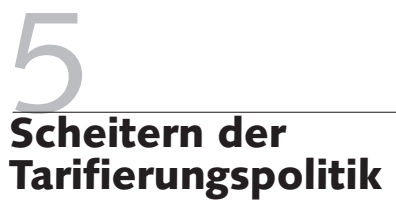

Infolge der anhaltenden Mitgliederschwäche konnten die Flächentarifverträge der Zeitarbeitsbranche auch in den nachfolgenden Tarifverhandlungen kaum weiterentwickelt werden, sodass sich die Entgeltunterschiede zwischen den Tariflöhnen der Leiharbeit und den Tarifen der Metall- und Elektroindustrie weiter vergrößert haben.

Alternative Ansätze der Einzelgewerkschaften und des DGB, auf juristischer Grundlage gegen den Christlichen Gewerkschaftsbund vorzugehen, beziehungsweise dessen Konkurrenz durch einen gesetzlich sanktionierten Branchenmindestlohn einzugrenzen, blieben bisher ohne Erfolg.

Das Scheitern der Tarifierungspolitik hat vor dem Hintergrund des rasanten Wachstums der Leiharbeit den Problemdruck im Organisationsbereich der IG Metall erheblich gesteigert. Besonders problematisch ist in diesem Zusammenhang ein wachsendes Segment von Betrieben, in denen Leiharbeit intensiv und über längere Zeitspannen eingesetzt wird. Nach den Ergebnissen des Trendreports Betriebsräte- wahlen 2006 gibt es im Organisationsbereich der IG Metall rund 700 Betriebe, in denen der Anteil der Leiharbeiter mit einer mindestens dreimonatigen Einsatzdauer mehr als $10 \%$ der Belegschaft beträgt. In über 100 Betrieben sind sogar mehr als die Hälfte der Belegschaft Leiharbeiter mit einer mindestens dreimonatigen Einsatzdauer (Rudolph/Wassermann 2007, S. 5).

In vielen Betrieben hat sich der Leiharbeitereinsatz damit zu einer Art „zweiten Belegschaft" entwickelt, die nicht nur einen hohen Konkurrenzdruck auf die Stammbelegschaften ausübt, sondern angesichts niedriger Organisationsgrade und eigener Tarifverträge häufig auch die gewerkschaftliche Arbeitskampffähigkeit in diesen sogenannten Intensivnutzerbetrieben infrage stellt. Vor diesen Hintergrund hat sich in der IG Metall eine zunehmend kritischere Haltung gegenüber der Leiharbeit ausgebreitet, die sich vor allem in Forderungen nach Eindämmung des Leiharbeitereinsatzes und Durchsetzung des Gleichbehandlungsgrundsatzes artikuliert (IG Metall 2007).

\subsection{KAMPAGNE „ZEITARBEIT MENSCHLICH“}

In einer ersten Reaktion auf den wachsenden Problemdruck startete der IG Metall Bezirk Berlin-Brandenburg-Sachsen im November 2006 die Kampagne „Zeitarbeit menschlich".

Vor dem Hintergrund eines überdurchschnittlichen Anteils von Leiharbeitsverhältnissen in Ostdeutschland wurde hier bereits Ende 2004 eine bezirkliche Projektgruppe zur Leiharbeit gegründet, die unter Einbeziehung lokaler Arbeitskreise zur Zeitarbeit und verschiedener Verwaltungsstellen Handlungskonzepte für die Leiharbeit entwickelte.

Zentraler Ansatzpunkt der Kampagne ist der Entleihbetrieb. Hier sollen über die gewerkschaftlichen Vertretungen in den Betrieben die Mitgliederwerbung und Interessenvertretung der Leiharbeiternehmer organisiert und betriebliche Regelungen zum Leiharbeitereinsatz erzielt werden. Auf diese Weise soll sowohl die im Vergleich zu den Verleihbetrieben günstigere Kontaktmöglichkeit in den Entleihbetrieben zur Mitgliederwerbung von Leiharbeitnehmern genutzt werden als auch auf den Handlungsdruck zur Begrenzung und Regulierung des Leiharbeitereinsatzes in den Entleihbetrieben reagiert werden.
Grundlage dieses Konzepts ist der Aufbau eines gewerkschaftlichen Betreuungssystems, dessen Kernbestandteil sogenannte Zeitarbeitsbeauftragte in den Entleihbetrieben sind. Bei den Zeitarbeitsbeauftragten handelt es sich in der Regel um Betriebsratsmitglieder, die durch themenbezogene Schulungen qualifiziert und innerhalb des Betriebsratsgremiums als Ansprechpersonen und Interessenvertretung für die eingesetzten Leiharbeitnehmer tätig sind. Die Mitgliedergewinnung von Leiharbeitern erfolgt darauf aufbauend im Kontext der betrieblichen Interessenvertretung und wird von Seiten der Projektleitung durch zielgruppenorientierte Werbematerialien unterstützt.

Ergänzend zum betrieblichen Betreuungskonzept wurde ein Servicetelefon eingerichtet, das organisierten Leiharbeitnehmern im Bedarfsfall Hilfestellung über die lokal zuständige Verwaltungsstelle oder den zuständigen Betriebsrat organisiert.

Ein zweiter Schwerpunkt der Kampagne ist die Initiierung und Unterstützung betriebspolitischer Ansätze zur Regulierung des Leiharbeitereinsatzes. Die angestrebten Regelungen zielen dabei zum einen auf die Angleichung der Arbeitsstandards von Stammbeschäftigten und Leiharbeitnehmern in Form von betrieblich vereinbarten Lohnzuschlägen sowie Gleichstellungen in Bezug auf betriebsbezogene Leistungen. Zum anderen wird versucht, den Leiharbeitereinsatz über entsprechende Regelungen zu begrenzen. Die Unterstützung dieser Initiativen erfolgt durch Beratung und Qualifizierung von Betriebsräten hinsichtlich der rechtlichen Einflussmöglichkeiten, daneben aber vor allem durch eine offensive Presse- und Öffentlichkeitsarbeit, die durch die Problematisierung und Skandalisierung der Arbeitssituation von Leiharbeitern in den Entleihbetrieben öffentlichen Druck auf die Arbeitgeber erzeugen soll.

Im Mittelpunkt der damit verbundenen Aktivitäten stand bisher die Veröffentlichung eines „Sozialreport Zeitarbeit“ im März 2007, der, ähnlich dem Schwarzbuch Lidl, mit Problemberichten von Leiharbeitern einige Resonanz in den Medien erlangte. Neben der Thematisierung von einzelnen betrieblichen Fällen soll die Öffentlichkeitsarbeit aber auch zu einer insgesamt kritischeren Wahrnehmung der Arbeitsbedingungen von Leiharbeitern beitragen und so sowohl innerhalb der Gewerkschaften als auch gegenüber der Politik Hand- 
lungsbedarf hinsichtlich der Regulierung von Leiharbeit vermitteln.

\subsection{KAMPAGNE "GLEICHE ARBEIT - GLEICHES GELD“}

Als zweites Projekt auf der Bezirkebene startete die IG Metall Nordrhein-Westfalen im April 2007 die Kampagne „Gleiche Arbeit-Gleiches Geld“. Ebenso wie bei der Kampagne „Zeitarbeit Menschlich“ besteht die Zielsetzung in der Etablierung einer neuen Betriebspolitik in den Entleihbetrieben, die neben der Mitgliederwerbung und Interessenvertretung von Leiharbeitern auch die betriebliche Regulierung des Leiharbeitereinsatzes beinhaltet. Das primäre Regulierungsziel besteht dabei neben der Begrenzung von Leiharbeit in der Umsetzung des Grundsatzes gleicher Bezahlung.

Zentrales Element zur Durchsetzung der betrieblichen Ziele ist hier allerdings die Umsetzung zeitlich begrenzter betriebspolitischer Kampagnen in den Entleihbetrieben, die von zwei Projektbeauftragten unterstützt werden. Im Rahmen dieser Kampagnen sollen auf der Basis der betrieblichen Bedingungen des Leiharbeitereinsatzes Forderungen an den Arbeitgeber entwickelt und gegebenenfalls über eine betriebliche Auseinandersetzung durchgesetzt werden. Zur Stärkung der betrieblichen Durchsetzungsmacht zielt der Prozess dabei auf eine Mobilisierung von Belegschaft und Leiharbeitnehmern im Rahmen von Informationsveranstaltungen und beteiligungsorientierten Aktionen für die Umsetzung der Forderungen. Die hauptamtliche Unterstützung bezieht sich im Vorfeld auf die Planung der Kampagne auf Grundlage einer gemeinsamen Recherche zum innerbetrieblichen Leiharbeitseinsatz und beinhaltet in der Konfliktphase die Unterstützung bei inner- und außerbetrieblichen Aktionen und Informationsveranstaltungen. Aktionsformen sind dabei etwa die kreative Gestaltung von themenbezogenen Betriebsversammlungen, die Sammlung von Unterschriften zur Unterstützung der Forderungen in den Entleihbetrieben, Bodenzeitungen und kostenlose Beratungsstunden für Leiharbeitnehmer vor den Einsatzbetrieben.

Wie im Bezirk Berlin-BrandenburgSachsen werden die betrieblichen Auseinandersetzungen durch eine kampagnenbegleitende Öffentlichkeitsarbeit unterstützt, die durch eine aktionsorientierte Presse- arbeit Druck auf den Arbeitgeber erzeugen, daneben aber auch zur Politisierung des Themas beitragen soll.

Insgesamt zielen diese Elemente auf eine erhöhte Durchsetzungsfähigkeit im betrieblichen Konflikt. Gleichzeitig werden die während der Auseinandersetzung stattfindenden Aktionen und Treffen aber von den haupt- und ehrenamtlichen Funktionären auch für die konfliktbezogene Werbung von neuen Mitgliedern unter den Leiharbeitnehmern genutzt.

\subsection{LEIPZIGER SIGNAL}

Im Anschluss an die Kampagnen in den Bezirken Berlin-Brandenburg-Sachsen und Nordrhein-Westfalen wurde auf dem Gewerkschaftstag der IG Metall im November 2007 die Initiierung einer bundesweiten Kampagne zur Leiharbeit beschlossen, die sich die Umsetzung des Gleichbezahlungsund Gleichbehandlungsgrundsatzes, die Eindämmung des Leiharbeitereinsatzes sowie die Erlangung eines aktionsfähigen Organisationsgrades der Leiharbeitnehmer zum Ziel gesetzt hat.

Ein Ansatzpunkt der bundesweiten Kampagne sind, wie in den Bezirkskampagnen, die Entleihbetriebe, über die die Mitgliedergewinnung und Regulierung des Leiharbeitereinsatzes organisiert werden soll. Daneben richtet sich die Kampagne aber auch an die Politik, von der eine gesetzliche Regulierung der Leiharbeit im Sinne der Kampagnenziele gefordert wird.

Den Kernbestandteil der Kampagne bildet die Politisierung des Themas in den Entleihbetrieben, den Organisationsgliederungen und der Öffentlichkeit. Auf diese Weise soll bei den Betriebsräten und Vertrauensleuten, aber auch bei der Politik, Problembewusstsein geschaffen werden, um die Umsetzung der Kampagnenziele zu befördern.

Weitere Bestanteile der Kampagne sind:

- die Erstellung von Materialien für Leiharbeitnehmer und betriebliche Funktionäre, unter anderem auch mit dem Ziel, ein Aktionshandbuch zur Planung und Umsetzung betrieblicher Kampagnen zu publizieren;

- die Qualifizierung von Hauptamtlichen, Betriebsräten und Vertrauensleuten zum Thema Leiharbeit im Rahmen von erweiterten Seminar- und Schulungsangeboten; - eine gezielte Öffentlichkeitsarbeit, mit der Druck für die Durchsetzung der ge- nannten Ziele gegenüber Arbeitgebern von Ent- und Verleihbetrieben geschaffen werden soll;

- die Einsetzung von Zeitarbeitsbeauftragten in den Entleihbetrieben zur Betreuung der Leiharbeitnehmer;

- eine bundesweite Mitgliederoffensive "Leiharbeit", mit der Leiharbeitnehmer systematisch für die Organisierung in der IG Metall gewonnen werden sollen.

Im Wesentlichen werden damit die Elemente der bezirklichen Kampagnen „Zeitarbeit menschlich“ und „Gleiche Arbeit Gleiches Geld“ aufgenommen und bundesweit verbreitet.

Darüber hinaus sollen im Rahmen der bundesweiten Kampagne aber auch die Internetplattform ZOOM sowie die lokalen Arbeitskreise zur Leiharbeit gefördert werden, um die Leiharbeit auch organisatorisch in der IG Metall zu verankern.

\section{Strategien in der Leiharbeit}

Mit Blick auf die eingangs genannten $\mathrm{He}-$ rausforderungen lassen sich drei verschiedene Strategieansätze erkennen:

\section{(1) Tarifierungsstrategie}

Die Akzeptanz von Leiharbeit durch die IG Metall nach der Abkehr von der Verbotsforderung war mit einem Ansatz verbunden, der sich als Tarifierungsstrategie kennzeichnen lässt. Im Vordergrund stand in dieser Zeit die Regulierung der Leiharbeit, die mit der Hoffnung auf eine „Normalisierung" der Leiharbeitsbranche einherging. Diese Strategie wurde zunächst durch das Interesse einzelner Arbeitgeber an der Anerkennung ihrer Zeitarbeitsfirmen durch die IG Metall, später dann durch das Interesse der Regierung an der Anerkennung der Leiharbeit durch die Gewerkschaften befördert. Bis auf einzelne Betriebsratsgründungen in der Zeitarbeitsbranche zu Beginn der Tarifierungsstrategie wurden keine besonderen Anstrengungen zur Mitgliedergewinnung bei Leiharbeitnehmern unternommen.

\section{(2) Partizipationsstrategie}

Mit der Gründung des ZOOM-Projekts und der Förderung lokaler Arbeitskreise erfolgte die organisationspolitische Öff- 
nung der IG Metall gegenüber der Leiharbeitnehmerschaft.

Um die Repräsentation von Leiharbeitnehmern in der IG Metall zu ermöglichen, wurden mit dem Projekt ZOOM und den Arbeitskreisen zur Leiharbeit partizipative Strukturen für die organisatorische Integration von Leiharbeitern geschaffen. In diesem Zusammenhang kann von einer Partizipationsstrategie gesprochen werden.

\section{(3) Kampagnenstrategie}

Die Initiierung der Kampagnen „Zeitarbeit Menschlich“ und „Gleiche Arbeit - Gleiches Geld“ kann demgegenüber als Kampagnenstrategie beschrieben werden. Die Strategie verfolgt dabei sowohl die Regulierung als auch Organisierung der Leiharbeit. Zentrales Mittel zur Durchsetzung der Forderungen ist die Aktivierung und Mobilisierung in den Entleihbetrieben im Rahmen einer konfliktorientierten Vorgehensweise. Gleichzeitig beinhaltet die Strategie die kampagnengeförderte Organisierung und Interessenvertretung der Leiharbeiter über die gewerkschaftliche Vertretung in den Entleihbetrieben.

Zusammenfassend lassen sich anhand dieser Entwicklung zwei Tendenzen erkennen: Erstens eine Tendenz von einer anwaltlichen Interessenvertretung der Leiharbeitnehmer zu einer verstärkt basisorientierten, konfliktbereiten Interessenvertretung von Stammbelegschaften und Leiharbeitnehmern. Diese spiegelt sich in den neuen Formen der organisatorischen und betrieblichen Integration der Leiharbeitnehmerinteressen wider, aber auch im Positionswandel von einer akzeptierenden zu einer wieder zunehmend kritischen Haltung gegenüber der Leiharbeit sowie in der erneuten Betonung des Gleichbehandlungsgrundsatzes.

Zweitens lässt sich eine Tendenz von der Orientierung an einer "Einflusslogik“ (Streeck 1999, S. 288) im Rahmen korporatistischer und sozialpartnerschaftlicher Arrangements hin zu einer zunehmenden Orientierung an gewerkschaftlicher Organisationsmacht erkennen. Diese Umorientierung äußert sich zum einen in der strategischen Neuausrichtung des Regulierungsansatzes von der branchenorientierten Tarifpolitik auf die organisationsstarken Entleihbetriebe, zum anderen in der Ausrichtung auf eine systematische Mitgliedergewinnung.
Die Neuausrichtung erfolgt dabei nicht allein im Rahmen konventioneller Formen gewerkschaftlicher Organisationsmacht, sondern geht mit einer Reihe neuer, für das duale System der Interessenvertretung eher ungewöhnlicher Praktiken der Organisierung, Interessenvertretung und -durchsetzung einher. Ein Element sind dabei die neuen Formen außerbetrieblicher Organisierung, wie sie sich in den beteiligungsorientierten Angeboten der Internetplattform ZOOM und den lokalen Arbeitskreisen zur Leiharbeit finden.

Ein zweites Element sind die unkonventionellen Formen betrieblicher Auseinandersetzung. Hierzu gehört eine skandalisierende Öffentlichkeitsarbeit, die betriebliche Problemstellungen mit Themen sozialer Gerechtigkeit verbindet, um auf diese Weise Druck auf die Arbeitgeberseite zu erzeugen, sowie inner- und außerbetriebliche Aktionen, die durch Einbindung der Beschäftigten den gewerkschaftlichen Forderungen Nachdruck verleihen sollen. Insgesamt kann in diesem Zusammenhang von einer Politisierung der Betriebspolitik gesprochen werden - im Gegensatz zu herkömmlichen Formen einer vorwiegend auf juristische Auseinandersetzung gerichteten Betriebspolitik.

Ein drittes Element ist die mit den Kampagnen verbundene „strategische Neuorientierung im Einsatz von Organisationsmacht" (Silver 2005, S. 141). Diese findet sich zum einen in dem strategischen Wechsel des Organisierungsansatzes von der Zeitarbeitsfirma zum Entleihbetrieb, mit dem Ziel, die dort bestehenden gewerkschaftlichen Strukturen für die Interessenvertretung und Mitgliedergewinnung von Leiharbeitnehmern zu nutzen; zum anderen im Wechsel des Regulierungsansatzes von der tarifvertraglichen Regulierung der Zeitarbeitsbranche zur betrieblichen Regulierung über die Entleihfirma, um die dort bestehende Organisationsmacht für die Interessendurchsetzung von Leiharbeitnehmern und Stammbelegschaft zu mobilisieren.

In Abgrenzung gegenüber der pfadkonformen Tarifstrategie verweisen diese Elemente der Partizipations- und Kampagnenstrategie angesichts neuer Herausforderungen für die Gewerkschaften auf eine zunehmende Bedeutung neuer, nicht institutionalisierter Formen der gewerkschaftlichen Organisierung und Interessenvertretung (AG Strategic Unionism 2007, S. 105).

\section{Ausblick}

Über die Wirksamkeit der neuen Ansätze lassen sich zu diesem Zeitpunkt noch keine abschließenden Aussagen machen, allerdings können auf Basis erster Ergebnisse bereits einige Probleme und Perspektiven identifiziert werden.

Mit Blick auf den Ansatz des ZOOMProjekts und der damit verbundenen lokalen Arbeitskreise resultieren Probleme vor allem aus dem außerbetrieblichen Charakter dieser Präsenzformen. Diese setzt hohe Hürden, einerseits hinsichtlich der Erreichbarkeit, andererseits hinsichtlich der Interessenvertretung, die sich weitgehend auf Beratungsleistungen und eine mittelbare Einflussnahme auf die gewerkschaftlichen Interessenvertretungen in den Entleihbetrieben beschränkt. Eine umfassende Mitgliederwerbung wird auf diesem Wege vermutlich nicht umsetzbar sein. Trotzdem ist es über diese Ansätze gelungen, ein bundesweites Netzwerk aktiver Leiharbeitnehmer und Betriebsräte zu etablieren und auf diese Weise die Leiharbeit organisatorisch und interessenpolitisch in der IG Metall zu verankern.

Gegenüber den lokalen Arbeitskreisen bietet die Kampagnenstrategie einen wesentlich breiteren strategischen Ansatzpunkt für die gewerkschaftliche Organisierung und Interessenvertretung in der Leiharbeit. Erste positive Ergebnisse aus einer ganzen Reihe von Betrieben zeigen, dass es hierdurch möglich ist, betriebliche Regelungen zu Lohnzuschlägen und Übernahmen von Leiharbeitnehmern durchzusetzen sowie betriebliche Vertreter für die Mitgliederwerbung zu aktivieren und beachtliche Erfolge bei der Organisierung von Leiharbeitnehmern zu erzielen.

Gleichzeitig lassen sich mit Blick auf die Reichweite der betrieblichen Umsetzung der Kampagnenstrategie aber auch einige Problemfelder identifizieren. Hier ist zum einen die in Konkurrenz zu der in den Kampagnen geforderten inklusiven Interessenvertretung stehende Orientierung vieler Betriebsratsgremien auf eine auf die Stammbelegschaft konzentrierte exklusive Interessenvertretung zu nennen. Zum anderen sind aber auch die mit der Kampagnenstrategie verbundenen neuen Anforderungen an die betriebliche Interessenvertretung durch die institutionellen 
Handlungsressourcen des Betriebsverfassungsgesetzes nur partiell abgedeckt. Dies gilt besonders im Hinblick auf die konfliktorientierten Handlungsansätze der Kampagnen, die nicht nur neue Formen der Interessendurchsetzung erfordern, sondern auch Reibungspunkte mit einer im System der dualen Interessenvertretung angelegten sozialpartnerschaftlichen Ausrichtung von Betriebsratsarbeit aufweisen.

Beide Problemfelder lassen darauf schließen, dass eine breite Verankerung der neuen Strategie vermutlich mit einem län- gerfristigen Prozess verbunden sein wird, der neben der Bereitstellung gewerkschaftlicher Hilfsangebote in vielen Fällen auch die Vermittlung eines - inklusive Konflikte nicht scheuenden - Verständnisses betrieblicher Interessenvertretungsarbeit erfordert.

\section{LITERATUR}

AG Strategic Unionism: Brinkmann, U./Choi, H./Detje, R./Dörre, K./ Holst, H./Karakayali, S./Schmalstieg, C. (2007): Strategic Unionism: Aus der Krise zur Erneuerung?, Umrisse eines Forschungsprogramms, Jena, Manuskript

Aust, A./Holst, H. (2006): Gewerkschaften und atypisch Beschäftigte. Von der Ignoranz zur Organisierung? Gewerkschaftliche Strategien im Umgang mit atypisch Beschäftigten am Beispiel von Callcentern und Leiharbeit, in: Industrielle Beziehungen 4, S. 291-313

Aust, A./Pernicka, S./Feigl-Heihs, M. (2007): "Moderner Sklavenhandel "?, in: Pernicka, S./Aust, A.: Die Unorganisierten gewinnen. Gewerkschaftliche Rekrutierung und Interessenvertretung atypisch Beschäftigter - ein deutsch-österreichischer Vergleich, Berlin, S. 231-312

Deutscher Gewerkschaftsbund (DGB) (2003): Ratgeber Zeitarbeit Handlungshilfe für Betriebs- und Personalräte, Berlin

Bundesagentur für Arbeit (2007): Arbeitsmarkt in Zahlen, Arbeitnehmerüberlassung, 2. Halbjahr 2006, Nürnberg

Dörre, K./Kraemer, K./Speidel, F. (2004): Prekäre Arbeit. Ursachen, soziale Auswirkungen und subjektive Verarbeitungsformen unsicherer Beschäftigungsverhältnisse, in: Das Argument 256, Hamburg, S. 378-397 IG Metall (2007): Handlungsansätze für ein tarif- und betriebspolitisches Konzept der IG Metall zur Arbeitnehmerüberlassung

Kommission Moderne Dienstleistungen am Arbeitsmarkt (2002): Moderne Dienstleistungen am Arbeitsmarkt. Vorschläge der Kommission zum Abbau der Arbeitslosigkeit und zur Umstrukturierung der Bundesanstalt für Arbeit, Berlin

Pernicka, S./Holst, H. (2007): Theoretische Perspektiven, in: Pernicka, S./Aust, A.: Die Unorganisierten gewinnen, Berlin, S. 231-312
Promberger, M./Bellmann,L./Dreher, C./Sowa, F./Schramm, S./Theuer, S. (2006): Leiharbeit im Betrieb. Strukturen, Kontexte und Handhabung einer atypischen Beschäftigungsform, Abschlussbericht, Nürnberg Rudolph, H. (2003): Befristete Arbeitsverträge und Zeitarbeit. Quantitäten und Strukturen „prekärer Beschäftigungsformen “, in: Linne, G./ Vogel, B. (Hrsg.): Leiharbeit und befristete Beschäftigung, S. 9-26 Rudolph, W./Wassermann, W. (2007): Gestärkte Betriebsräte, Trendreport Betriebsrätewahlen 2006, Ergebnisse der erweiterten Analyse, Kassel Schlese, M./Schramm, F. (2004): Implikationen der Tarifverträge zur Leiharbeit für die Tarif- und Beschäftigungsbedingungen im GebäudereinigerHandwerk, Berlin, Hamburg

Schüren, P. (2005): Aktuelle Rechtliche Probleme der Zeitarbeit, in: Beck, D. (Hrsg.): Zeitarbeit als Betriebsratsaufgabe, Düsseldorf, S. 11-22

Silver, B. J. (2005): Forces of Labor. Arbeiterbewegungen und Globalisierung seit 1870, Berlin, Hamburg

Streeck, W. (1999): Staat und Verbände: Neue Frage, neue Antworten?, in: Streeck, W.: Korporatismus in Deutschland. Zwischen Nationalstaat und Europäischer Union, Frankfurt/Main, New York Ulber, J. (2004): Arbeitnehmer in Zeitarbeitsfirmen, Frankfurt/Main Wassermann, W./Rudoph, W. (2007): Leiharbeit als Gegenstand betrieblicher Mitbestimmung. Anforderungen und Arbeitsressourcen von Betriebsräten in Betrieben mit hohem Leiharbeitnehmeranteil hrsg. von der Hans-Böckler-Stiftung, Düsseldorf

Weinkopf, C. (2006): Mindestbedingungen für die Zeitarbeitsbranche?, Expertise im Auftrag des Interessenverbandes Deutscher Zeitarbeitsunternehmen (iGZ e. V.), Gelsenkirchen 